\title{
GANGRENE IN CONGENITAL SYPHILIS
}

\author{
BY
}

W. P. FOSTER, M.B., Ch.B., and R. G. G. TAYLOR, M.B., Ch.B.

(From the Department of Child Health, University of Bristol, and the Children's Department of the United Bristol Hospitals)

Peripheral circulatory disorder in early childhood is somewhat unusual except as a temporary disturbance consequent upon capillary stagnation, coldness, or other simple reversible phenomena. The occurrence of ischaemic disorder sufficient to evoke gangrenous changes is not only rare, but is clinically striking and necessitates an urgent approach to the etiology.

Acute arteritis may occur during or subsequent to severe infection. Such arterial changes have been recorded in the enteric fevers, typhoid fever, scarlet fever, and diphtheria. In the newborn, general or umbilical sepsis has been related to acute arterial ischaemic necrosis, especially in the limbs. In most examples the terminal branches of the limb circulatory system have become variably affected with arterial thrombosis to an extent sufficient to precipitate rapid necrosis of even a hand or a foot. Under these circumstances it is reasonable to suppose that the arterial walls have succumbed to a focal nodular, or more diffuse, necrotizing arteritis, and sudden massive endarterial occlusion has supervened. In some cases arterial embolism may be the operative cause, but this is much less likely in view of the wide lability of possible collateral routes of blood supply of the limbs in the young child.

Many of the clinical effects of infection with the Treponema pallidum are related to the endarterial changes in the individual focal lesions, especially in the acquired forms of syphilis. One of the outstanding features of congenital syphilis, however, is the peculiar insusceptibility of the cardiovascular system and a relative immunity of the arterial structures to direct disease or degeneration. It has, however, been occasionally observed that ischaemic effects, with in some cases an extension to tissue necrosis, may take place and are more usual in the young child. Illustrated examples of this condition appear in the works of Stokes (1944), and Pritchard (1938). Others have noted an occasional clinical relationship between congenital syphilis, peripheral arterial disease, and paroxysmal haemoglobinuria: in such a group disorder the phasic haemoglobinuria may arise proportionately to the cooling effect and local circulatory stagnation consequent upon the syphilitic endarterial disease.

Whilst it must be recognized that vascular lesions of such severity are only remotely probable, the example recorded here is of special interest in view of the extensive and crippling damage which ensued and of the rapid and satisfactory remedial effects obtained in some peripheral areas which were threatened with gross destructive changes.

\section{Case History}

A.B., aged 2 years, was the second child in the family. The first child, a boy aged 7 years, was healthy and had a negative Wassermann test. A third child died at the age of 1 month from alleged bronchopneumonia. The parents appeared to be well and gave no history of syphilitic lesions.

A.B. first came under medical observation in hospital on Feb. 18, 1948, at the age of 14 months. He was a full-term infant of normal delivery and appearance, but there was a history of a rash on the nates and some troublesome rhinitis during the earlier weeks of life. Progress had not been satisfactory, and admission to a regional hospital was sought because the baby was miserable, listless, and of inadequate weight. The admitting physician noted that there were some clinical features of unusual significance, and a special record was made in regard to rhinitis, some 'bossing ${ }^{-}$of the frontal region of the head, and a rash irregularly disposed over the face and buttocks. The skin was generally dry and the hair sparse. There was no evidence of any visceral abnormality and the spleen and liver were not enlarged. The nervous system, including the eyes and hearing, was normal. The right hand was cyanosed and oedematous, and the tips of the second and third fingers were discoloured and tender. On the left hand the tips of the third, fourth, and fifth fingers were blue and tender (fig. 1). The right foot showed a necrotic area on the dorsum and the fourth toe was discoloured, but these variably affected areas were not recognizably cold to touch (fig. 2). Careful examination failed to reveal any significant reduction in pulsation of the radial and dorsalis pedis arteries on either side. During the following week the darkening discolouration of the fingers, especially of the larger part of the right hand (fig. 3), became suggestive of a gangrenous transformation, and the affected parts 
failed at any time, even under conditions of applied warmth, to improve. Vasodilator drugs also failed to relieve the local circulatory disturbance. It was noted that the affected areas were warmer to palpation than their appearance indicated. As a means of forestalling secondary sepsis, a course of sodium penicillin $(50,000$ units six-hourly intramuscularly) was begun. The right hand remained darkened and became very swollen and it appeared that a red line of demarcation was developing. Amputation was suggested, but this was considered unwise owing to the poor general condition of the child. Shortly afterwards the child's blood Wassermann reaction was reported strongly positive, and the same result was obtained in both parents. In view of this, penicillin was continued and was augmented by injections of bismuth $(0 \cdot 25 \mathrm{ml}$. daily) and suitable doses of potassium iodide and liquor hydrarg. perchlor. The child improved clinically during the following few weeks, but unfortunately the nutritional state of the right hand failed to improve proportionally. In fact dry gangrene supervened, with its limitations at the more defined line of demarcation. However, it seemed clear that the active gangrenous zone was considerably smaller than had appeared probable at an earlier date, especially before the commencement of urgent and intensive anti-specific therapy. It is of some interest that after the beginning of penicillin treatment the child had had fever reaching to $103^{\circ} \mathrm{F}$.

A month later, during which time there had been difficulties about continuity of treatment and dangers had again arisen in regard to the gangrenous areas, the child entered the Bristol Children's Hospital on Feb. 22, 1948.

The outstanding clinical features at this admission were the characteristic 'special facies' with suspicious perioral scarring and a conspicuous facial profile. The dry gangrene of the right hand was sharply demarcated at the level of the proximal third of the metacarpus (fig. 4). The third, fourth, and fifth fingers of the left hand were plum-coloured, except for their tips which were quite black, and the nails appeared to be necrotic. The terminal phalanx on the fourth right toe was similarly blackened and the nail necrosed. No appreciable reduction of pulsation in the peripheral arteries could be detected. X-ray examination of the whole skeleton revealed an advanced and extensive degree of syphilitic osteo-periostitis with involvement of the tibial, femoral, and ulnar bones. Treatment with penicillin was again begun $(60,000$ units threehourly). On no occasion was haemoglobinuria detected, and the urine was repeatedly normal.

After one week's intensive penicillin treatment it was decided by Prof. R. Milnes-Walker that there was no alternative to amputation of the gangrenous portion of the right hand, and on March 30, 1948, this was carried out at the level of the metacarpophalangeal joint of the thumb and the metacarpal line of the remaining parts of the hand. At this time the child's general condition, especially the nasal infection, had considerably improved. Progress in the amputated area was satisfactory (fig. 5). A small eschar separated from the right fourth toe and the discoloured necrotic areas on the left hand showed corresponding marked improvement. The necrotic nails had separated. Two weeks after the surgical treatment the amputation area was granulating and healing satisfactorily. A course of three weeks' penicillin treatment was concluded. The blood Wassermann and Kahn reactions remained positive. Continuity of therapy was maintained by injections of sulpharsenobenzine as 'sulphostab' $0.01 \mathrm{~g}$. weekly and bismuth $0.01 \mathrm{~g}$. bi-weekly. It was clear that the anti-syphilitic treatment was proving effective, and excellent general improvement was apparent in nutrition, mental and physical activity, and a restoration of normal rate of growth.

On no occasion had the cerebrospinal fluid shown any abnormality, and it was perhaps remarkable, in view of the severe general infection, that the fluid's Wassermann reaction and the colloidal gold curves were normal.

Careful and continuous chemotherapy was maintained in the Children's Hospital over a period of twelve weeks. In the final period all signs of active disease had cleared and the Wassermann reaction became negative. Suitable continuity of treatment in the out-patient department was maintained.

Discussion
Peripheral vascular changes resulting in gangrene in a case of syphilis present, according to Stokes (1944) and other writers, little that is characteristic of syphilis. However, it is generally assumed that where the condition occurs in the presence of florid manifestations of syphilis this disease is responsible for the vascular changes which precede occlusion.

The possible causes of peripheral gangrene occurring in childhood are much less numerous than in adults. In adults many diseases must be excluded before syphilis can be diagnosed.

In both adults and children the only infallible method of ensuring the veracity of such a diagnosis would be the demonstration of the Treponema pallidum in the affected tissues. The literature does not afford very conclusive evidence that this has been done in the case of peripheral lesions. However, Warthin (1922) claimed to have found spirochaetes in popliteal and tibial arteries in symmetrical gangrene simulating Raynaud's disease.

In the case here reported, on the score of age, arteriosclerosis and von Buerger's disease could be eliminated as causes. From the history there was nothing to suggest a traumatic or toxic origin. Clinical findings in the child included a normal urine, and no evidence of cardiac abnormality. Diabetes and arterial embolism could also be excluded. Periarteritis nodosa, Raynaud's disease, and syphilis remained as possible causes.

Periarteritis nodosa may affect individuals of all ages, and the condition has been observed in infancy. Those cases which have shown digital gangrene from 


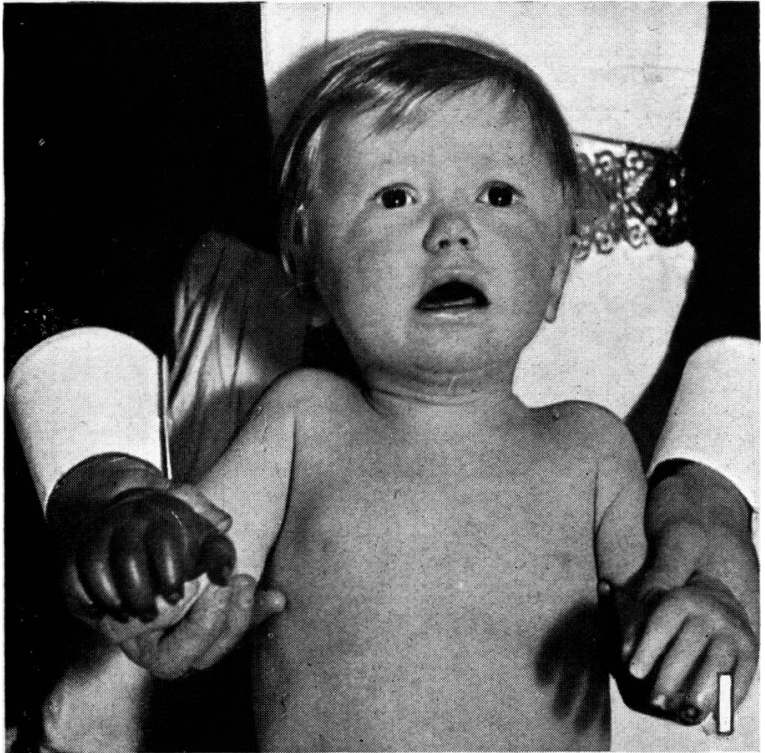

FIG. 1.-Condition shortly after admission (Feb. 18, 1948) showing facies and gangrene of hands. The photograph was taken on Feb. 25.

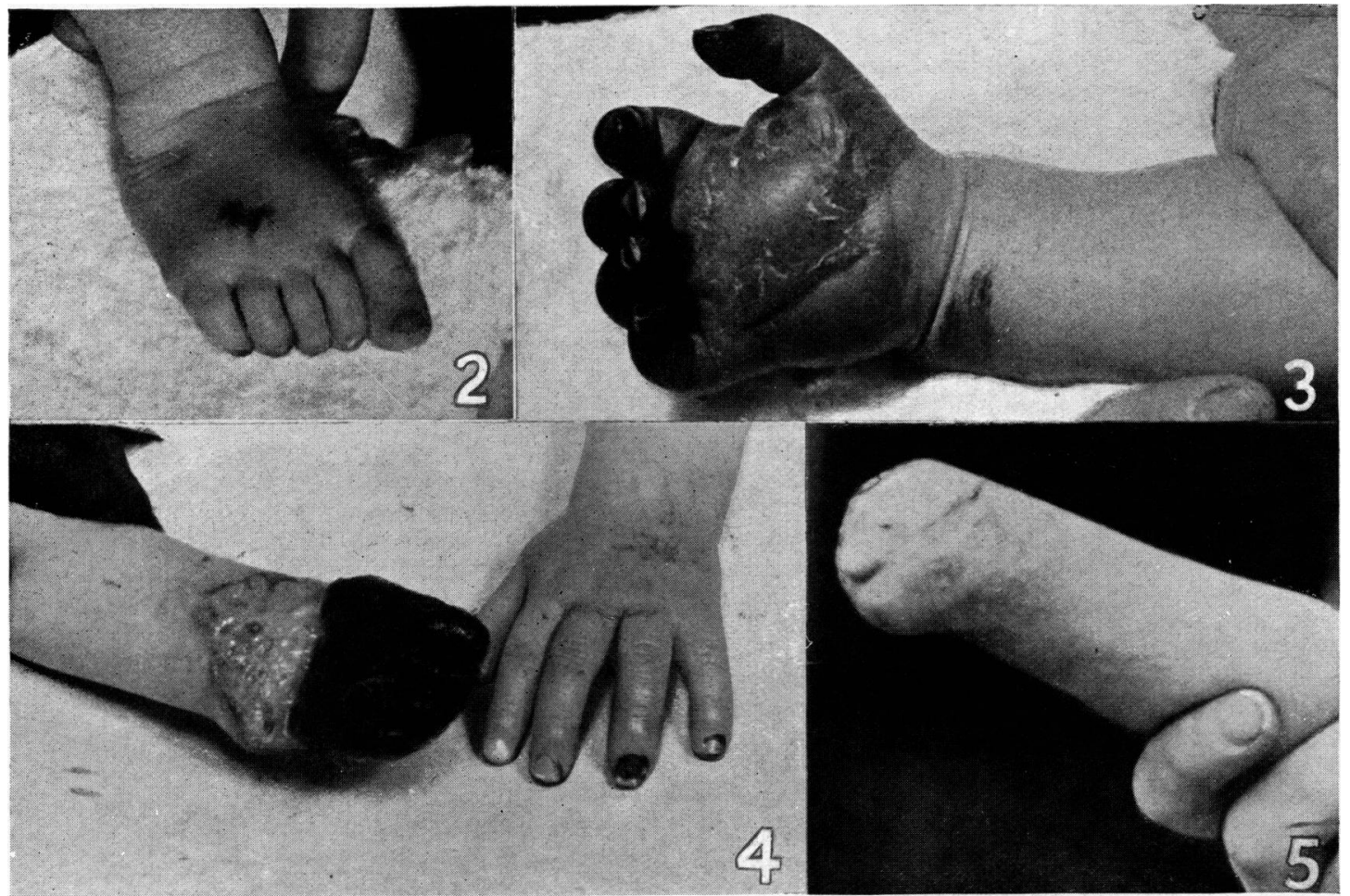

FIG. 2.-The right foot, showing necrotic area on the

FIG. 3.-Gangrene of right hand, February, 1948. dorsum (Feb. 25).

Fig. 4.-Hands, March 25, 1948, after the first course of penicillin. Note the line of demarcation of the gangrenous area.

Fig. 5.-Right upper limb one month later after excisionamputation of necrotic tissue (March 30). 
thrombotic occlusion of the peripheral arteries have also shown other constitutional effects and more widespread lesions. The more general symptoms and signs associated with periarteritis nodosa were noticeably absent in this case.

Raynaud's vascular disorder may occur at any age but is quite uncommon before puberty, and in order to constitute the clinical diagnosis the following criteria are usually required: (1) intermittent attacks of cyanosis and digital pallor, influenced by cold and emotion, the affected parts subsequently recovering with hyperaemia; (2) little or no change in the peripheral arterial pulses; (3) gangrene never presents at the first attack, and if it occurs is usually slight and confined to small areas only.

The case now discussed cannot be said to conform entirely to all of these criteria; indeed it is only in the matter, albeit an important one, of continued presence of arterial pulses that there is a resemblance to the Raynaud syndrome.

There exists a group of conditions in which paroxysmal digital cyanosis occurs to which the term 'Raynaud's phenomenon' is better applied. In such conditions the paroxysm is considered to be secondary to other bodily disease. There may be such digital cyanosis, sometimes progressing to gangrene, occurring during exposure to cold. Paroxysmal haemoglobinuria and, more rarely, severe urticarial phenomena, may be associated. This condition has been described with congenital syphilis in childhood. It is supposed that chilling of the endothelial cells of the smallest blood vessels favours arteriolar thrombosis in the parts affected. Where syphilis is present energetic anti-syphilitic treatment offers a prospect of recovery.

The case described here showed no evidence of urticaria on exposure to cold, and paroxysmal haemoglobinuria was not an apparent feature.

Where the effect of cold cannot be incriminated, then it is necessary to consider a syphilitic process as the cause of damage to the arterial wall. Syphilis may produce a panarteritis of which intimal hyperplasia is a principal feature. If this is sufficiently severe the circulation will be locally reduced and thrombosis may be expected. Possibly there is an initial period of peripheral ischaemia related to a reflex vasospastic response in the local inflammation. On such a hypothesis one might explain some of the beneficial effects of specific treatment in these cases.

Heller and Alvari (1941), in reviewing gangrene of the extremities in the newborn, assembled forty cases from the literature. Of these they were able to identify those due to pressure associated with difficult labour, those due to infection, and a number of cases vaguely attributed by the authors to 'Raynaud's disease' or neurological disorder. They advanced an ingenious theory of temporary agglutination of the intimal surfaces of the smaller arteries, in which fibrin sealed the surfaces together but in which thrombosis formation did not necessarily occur. Removal of pressure or spasm would allow the pulsating proximal blood column to wedge open the sealed walls and so restore the circulation before an irreversible change occurred. These authors plead for delay of amputation in such cases to see if there might be any improvement.

Sections of the excised necrotic tissue reveaked no change in the vascular walls that could be said to be characteristic of syphilis. This would agree with Stokes's experience. There was no evidence of any gummatous change in the vessel walls; such changes have, however, been recorded in adults with acquired specific disease.

In adults gangrene of the extremities is sometimes seen after acute infections, especially in the tropics. Bloss (1948) described such a case in an African, and referred to articles by Gelfand (1947) and Salter (1947), who have reported six cases, four of whom also had syphilis. A similar example was seen by one of us in Abyssinia. This patient had an acute attack of malaria and had syphilis as well. It would seem that syphilitic disease may well be a dominant factor in gangrene of the extremities following acute intercurrent disease.

In children and young adults bilateral gangrene of the extremities has been observed in the absence of obvious cause in apparently healthy individuals. More often there is a history of acute infection or preceding malnutrition. In such cases the changes in the affected parts is acute and proceeds quickly in the course of a few days. Some recovery, however, is usual, and the ultimate area of gangrene may be less than was originally threatened. The ears and nose may also be affected. Exposure to cold may not necessarily be an aggravating factor, and the parts are uninfluenced by heating. The pulses usually remain normal. It is interesting to note that Raynaud himself observed such a case. This was a boy of $3 \frac{1}{2}$ years, in whom the circulatory and tissue changes were attributed to emaciation.

Von Khantz (quoted by Heller and Alvari, 1941), in reporting fifty cases of gangrene of the extremities in childhood, attributed thirty nine of these to acute infection. The nature of known infections was distributed as follows: diphtheria 6; scarlet fever 8 ; measles 4 ; typhoid 6; syphilis 8 ; enteritis 4 ; typhus 3 ; pneumonia 2; omphalitis 2 . Our case has much in common with the clinical picture seen in this series, and yet syphilis was present.

Congenital syphilis is well known as a cause of failure to thrive and of malnutrition. The child in question was certainly malnourished at the time of recognition of the syphilitic disease. The lesions and the general health of the child improved considerably with anti-syphilitic measures, and it would appear that the gangrene here was due to a devascularizing effect of syphilitic infection rather than to general malnutrition consequent upon congenital syphilis.

Anwyl-Davies, Nabarro, and Parkes Weber (1940) reported gangrene of the extremities in two sisters. The children were the daughters of a woman who was herself a congenital syphilitic. Both children, as infants, had gangrene of the identical digits of 
the right hand. In both the lesions healed by scarring and tissue loss.

Treatment. Treatment with sodium penicillin initiated marked general improvement and obvious clinical retrogression of the local lesions. In the initial stages of the condition the child also received potassium iodide. The use of potassium iodide as a therapeutic test is, however, according to Stokes, of little value, as other vascular diseases besides those associated with syphilis may show some favourable response. The child received 50,000 units of sodium penicillin, six-hourly at first, to a total of $1,500,000$ units. Subsequently he received a further course of 60,000 units three-hourly for sixteen days.

We must admit the possibility that the initial treatment with penicillin may have been somewhat inadequate. Tissue necrosis was already incipient when treatment was begun, but in view of the extent of recovery, particularly as regards the digits of the left hand, more heroic doses might have been given with advantage.

In cases where syphilis is suspected early, energetic treatment at the stage of primary discoloration may possibly produce a recovery without loss of tissue. Barker (1948), reviewing penicillin treatment of syphilis, considers that the course of penicillin given for the treatment of congenital syphilis in the past tended to be inadequate and of insufficient duration, and the relapse rate was unfortunately high. It appears that the minimum dose should be 20,000 units per lb. of body weight, given three-hourly for fifteen days. The optimum dose may be even larger and should be about 50,000 units in twenty-four hours per $l b$. of body weight, and given at threehourly intervals. In forty to fifty per cent. of cases a reaction in the form of mild fever for two or three days may follow commencement of penicillin. Such a reaction was shown in this child. Gastrointestinal disturbance may also occur in this phase of the therapy, but such reactions are rarely severe enough to justify any modification of the treatment.

\section{Summary}

An example of peripheral gangrene in a child under two years of age and suffering from congenital syphilis is described. Gangrene in the extremities in infancy and childhood is also briefly reviewed.

We are indebted to Prof. A. V. Neale for his interest in our paper and for his permission to use the clinical data. Dr. A. D. Fraser kindly reported upon the pathological examination of the excised tissue. We are grateful to the staff of the Dorset County Hospital for some of the earlier notes in the clinical history and for the photographs of the child.

\section{REFERENCES}

Anwyl-Davies, T., Nabarro, D., and Parkes Weber, F. (1940). Brit. J. Child. Dis., 37, 173.

Barker, L. P. (1948). J. Pediat., 32, 561.

Bloss, J. F. E. (1948). Brit. med. J., $2,52$.

Gelfand, M. (1947). Ibid., 1, 847.

Heller, G., and Alvari, G. (1941). Amer. J. Dis. Child., 62, 133.

Herrmann, L. A. (1933). Amer. J. Syph., 17, 305.

Learmonth, G. E. (1925). Canad. med. Ass. J., 15, 69.

Lewis, T. (1936). Vascular Disorders of the Limbs. London. p. 77.

Lewis, T., and Pickering, G. W. (1934). Clin. Sci., 1, 327.

Moynahan, E. J. (1948). Brit. J. vener. Dis., 24, 104.

Pritchard, E. (1938). The Infant. London. p. 301.

Richards, R. L. (1946). 'Peripheral Circulation in Health and Disease. Edinburgh.

Salter. J. G. (1947). Brit. med. J., 3, 108.

Slaughter, W. H. (1926). J. Amer. med. Ass., 86, 1607.

Stokes. J. G.. Beerman, H., and Ingraham, N. R. (1944). Modern Clinical Syphiblogy. Philadelphia. p. 103.

Warthin, A. S. (1922). N.V. med. J., 115, 69.

\section{REVIEWS}

Garrod, Batten, and Thursfield's Diseases of Children (Fourth Edition). Edited by Donald Paterson, M.D., F.R.C.P., Consulting Physician, Hospital for Sick Children, Great Ormond Street, and AlaN MonCreIFF, M.D., F.R.C.P., Nuffield Professor of Child Health, University of London. Vol. II. With contributions by twenty-four contributors. 1949. London: Edward Arnold and Company. Pp. 1033. (Price 40s.)

The second volume of this famous textbook has appeared after the first volume has already been reprinted. This alone should ensure the successor an immediate and hungry public. The subjects dealt with are organic and functional disorders of the nervous system; congenital mental defect; diseases of the eyes, muscles, bones, and joints; orthopaedic surgery; medical diseases and surgery of the urogenital system; diseases of the liver, peritoneum, skin, and cardiovascular system; rheumatism; blood disorders; infections and venereal diseases; and malignant disease in childhood. The first section (organic diseases of the nervous system) is of particular interest, since it represents a combined production by a physician and a neurosurgeon; it has been entirely re-written, providing a reminder, perhaps, that neurology is not the sterile and static academic discipline sometimes imagined. With such variety of subjects and authors represented, it need only be said of the second volume as a whole that it maintains the standard 